\title{
GS263-02+45: a new HI supershell with evidence of having triggered star formation?
}

\author{
E. M. Arnal ${ }^{1,2, \star}$ and M. Corti ${ }^{2,3, \star \star}$ \\ 1 Instituto Argentino de Radioastronomía (IAR), C.C. No. 5, 1894 Villa Elisa, Argentina \\ e-mail: arnal@iar.unlp.edu.ar \\ 2 Facultad de Ciencias Astronómicas y Geofísicas, Universidad Nacional de La Plata, Paseo del Bosque s/n, 1900 La Plata, Argentina \\ 3 Instituto de Astrofísica de La Plata (IALP), CONICET, Argentina
}

Received 7 May 2007 / Accepted 17 August 2007

\begin{abstract}
Aims. We look for galactic neutral hydrogen (HI) supershells likely to be associated with massive star groupings. Methods. A neutral hydrogen (HI) line survey carried out at the Instituto Argentino de Radioastronomía (IAR) and the Southern Galactic Plane Survey (SGPS) were the main databases used in this investigation.

Results. A new HI supershell (GS263-02+45) was found in the outer part of the Galaxy. This structure is located at a distance of $5.3 \pm 1 \mathrm{kpc}$ from the Sun and has a linear diameter of about $665 \pm 40 \mathrm{pc}$. The expansion velocity of the supershell is $\sim 14 \mathrm{~km} \mathrm{~s}^{-1}$. The total amount of atomic gas related to GS263-02+45 is $\sim 2 \times 10^{6} M_{\odot}$. Based on the analysis of the SGPS HI data it is concluded that the OB-association Bochum 7 is likely to be interacting with GS263-02+45. This fact together with distance and age arguments may indicate that Bochum 7 was born as a consequence of the evolution of GS263-02+45.
\end{abstract}

Key words. Galaxy: kinematics and dynamics - Galaxy: structure - ISM: bubbles - ISM: structure - radio lines: ISM

\section{Introduction}

Since the pioneering work of Heiles $(1979,1984)$ it has been recognized that some of the most spectacular phenomena of the interstellar medium (ISM) are the so-called neutral hydrogen (HI) shells. These features, whose physical dimensions lie in the range from a few tens of parsecs to a few kiloparsecs (Rand \& Stone 1996; de Blok \& Walter 2000), are structures identified in the distribution of the HI in the Milky Way and in many nearby galaxies. Shells are usually identified, in a given velocity range, as a minimum in the HI distribution that is surrounded by walls of HI emission.

It is thought that most of the HI shells, especially the small ones, may be formed by stellar winds or supernovae, or their combined effects. However, the origin of the larger shells, or supershells, is far from being understood. They seem to require large ( $\left.\geq 10^{52} \mathrm{erg}\right)$ formation energies in order to maintain expansion velocities of tens of $\mathrm{km} \mathrm{s}^{-1}$ and to achieve dimensions of a few hundred parsecs (Heiles 1984). In order to power the most energetic supershells an unreasonably large number of massive stars, ranging from hundreds to thousands, are required. For these extreme cases, energetic events connected to a gamma-ray burst (Perna \& Raymond 2000), or the infall of a high velocity cloud into the HI disc (Tenorio-Tagle 1981), are thought to be alternative processes that may be at work to create these large structures.

Although the shocks associated with the expanding walls of the supershells may be a mechanism for triggering the formation of a new generation of stars, only a few examples are known (Oey et al. 2005). Star formation associated with

\footnotetext{
* Member of Carrera del Investigador Científico, CONICET.

$\star \star$ Fellow from CONICET, Argentina.
}

HI supershells are observed in the Large Magellanic Cloud (Kim et al. 1999) and in the Small Magellanic Cloud (Stanimirovic et al. 1999). Recently, several large-scale HI surveys (Taylor 1999; McClure-Griffiths et al. 2001) have increased the number of shells and supershells known to exist in the Galaxy. Based on the Southern Galactic Plane Survey (SGPS), McClure-Griffiths et al. (2002) reported the discovery of 19 new HI shells and supershells. Among the newly discovered objects they mentioned GSH263+00+47. Following the standard nomenclature the centre of this supershell is located at $(l, b)=\left(263^{\circ} .0,0^{\circ} 0\right)$ and its central velocity ${ }^{1}$ is $47 \mathrm{~km} \mathrm{~s}^{-1}$. The distance of GS263+00+47 is $5.5 \pm 1 \mathrm{kpc}$ (McClure-Griffiths et al. 2002). Recently, a new OB-association (Bochum 7) was discovered at $(l, b)=\left(265^{\circ} .0\right.$, -2.0 ) (Corti 2005). This association is located at a distance $4.5 \pm 0.4 \mathrm{kpc}$ (Corti 2005). The mean radial velocity and age of this stellar aggregate are $V=49 \pm 6 \mathrm{~km} \mathrm{~s}^{-1}$ and $(2-7) \times 10^{6} \mathrm{yr}$ (Corti et al. 2003).

As GSH263+00+47 and Bochum 7 have similar distances and radial velocities, and the latter being projected onto the outer border of GSH263+00+47, a physical link between both objects is possible. To analyse this possibility, we studied in more detail the HI distribution in a large area around GS263+00+47. The databases used in this study are briefly outlined in Sect. 2, the observational results are reported in Sect. 3, and the discussion and conclusions follow in Sects. 4 and 5.

\section{Databases}

The large region delimited by $255^{\circ} \leq l \leq 270^{\circ}$ and $-8^{\circ} \leq b \leq 5^{\circ}$ was reanalysed using the new high sensitivity IAR HI survey of

\footnotetext{
${ }^{1}$ All radial velocities are referred to the Local Standard of Rest (LSR).
} 
the southern sky. Arnal et al. (2000) describe the motivation and observing strategy of the IAR HI survey, Bajaja et al. (2005) describe the stray-radiation correction applied to the data, and Kalberla et al. (2005) present the data, combined with the northern material of the Leiden/Dwingeloo Survey (LDS).

The HI survey was carried out using a $30 \mathrm{~m}$ dish $\left(H P B W \sim 30^{\prime}\right)$ on a $0.5 / \cos b \times 0.5$ grid in galactic coordinates. The survey covers the velocity interval -400 to $450 \mathrm{~km} \mathrm{~s}^{-1}$, and the velocity resolution is $\sim 1.3 \mathrm{~km} \mathrm{~s}^{-1}$. The final $\mathrm{rms}$ noise of a single profile is $\sim 70 \mathrm{mK}$ on the brightness temperature scale. This scale is accurate to $5 \%$. The stray-radiation corrected version of the Hi survey (see Bajaja et al. 2005, for details) has been used in this paper.

A smaller region, $263^{\circ} \leq l \leq 268^{\circ}$ and $-5^{\circ} \leq b \leq 1^{\circ}$, was analysed making use of the SGPS (McClure-Griffiths et al. 2001). These data were retrived from the ATCA site. Infrared data from the Midcourse Space Experiment (MSX) (Price et al. 2001) were retrieved from the Infrared Science Archive ${ }^{2}$.

\section{Results}

Given the central radial velocity $\left(V_{\mathrm{o}} \simeq 47 \mathrm{~km} \mathrm{~s}^{-1}\right)$ and the expansion velocity $\left(V_{\exp } \simeq 14 \mathrm{~km} \mathrm{~s}^{-1}\right)$ of $\mathrm{GSH} 263+00+47$ (McClure-Griffiths et al. 2002), a large velocity range (from 20 to $70 \mathrm{~km} \mathrm{~s}^{-1}$ ) around $V_{\mathrm{o}}$ was inspected. Using the full velocity resolution of the IAR HI survey, the HI brightness temperature distribution spanning the velocity range mentioned above was thoroughly studied within the region delimited by $255^{\circ} \leq l \leq 270^{\circ}$ and $-8^{\circ} \leq b \leq+5^{\circ}$. To illustrate the main observational findings, in Fig. 1 a mosaic of twelve HI images covering the velocity range from $\sim 31.3$ to $\sim 55.7 \mathrm{~km} \mathrm{~s}^{-1}$ is shown. At $44.5 \mathrm{~km} \mathrm{~s}^{-1}$ (Fig. 1g) and $46.5 \mathrm{~km} \mathrm{~s}^{-1}$ (Fig. 1h) the HI brightness temperature images show the unmistakable signature of a large HI shell. The emission arising from this shell is far from uniform. In these two images the HI shell can be traced between $258^{\circ} \leq$ $l \leq 266^{\circ}$ and $-6^{\circ} \leq b \leq+2^{\circ}$. At lower radial velocities, the ring of HI emission characteristic of an expanding shell remains recognizable until $38.5 \mathrm{~km} \mathrm{~s}^{-1}$ (Fig. 1d). At $36.5 \mathrm{~km} \mathrm{~s}^{-1}$ (Fig. 1c) the shell-like feature is barely visible and at velocities lower than $36.5 \mathrm{~km} \mathrm{~s}^{-1}$ (Fig. 1c) it is difficult to recognize any trace of the HI shell. Towards the other velocity extreme (more positive) the large shell can be observed until $50.5 \mathrm{~km} \mathrm{~s}^{-1}$ (Fig. 1j), but from there onwards it becomes increasingly difficult to identify the HI features that may be related to it. This large HI shell will be designated as Feature A. Another HI structure is a small (with respect to Feature A) shell centred at $(l, b)=\left(263^{\circ}, 0^{\circ}\right)$. This shell is clearly seen at $46.5 \mathrm{~km} \mathrm{~s}^{-1}$ (Fig. 1h), $48.5 \mathrm{~km} \mathrm{~s}^{-1}$ (Fig. 1i) and $50.5 \mathrm{~km} \mathrm{~s}^{-1}$ (Fig. 1j). This feature covers the region $260^{\circ} \leq l \leq 266^{\circ}$ and $-2^{\circ} \leq b \leq+2^{\circ}$ and will be labelled Feature B. Based on its morphology and velocity extent, Feature B is identified as the shell GSH263+00+47 discovered by McClure-Griffiths et al. (2002).

In summary, along the velocity range 20 to $70 \mathrm{~km} \mathrm{~s}^{-1}$ two shell-like features were found in the HI distribution. One of them (Feature B) very likely corresponds to the shell GSH263+00+47 first identified by McClure-Griffiths et al. (2002), while the other (Feature A) is a much larger structure not reported before in the literature.

${ }^{2}$ The NASA/IPAC Infrared Science Archive is operated by the Jet Propulsion Laboratory, California Institute of Technology, under contract with the National Aeronautics and Space Administration. (http://irsa.ipac.caltech.edu)
The mean brightness temperature distribution in the range $39 \mathrm{~km} \mathrm{~s}^{-1}$ to $49 \mathrm{~km} \mathrm{~s}^{-1}$ is shown in Fig. 2. There the shell GSH263+00+47 reported by McClure-Griffiths et al. (2002) is shown as a broken ellipse, while the approximate boundary of the new HI shell is depicted by a solid line. Following the standard nomenclature this new shell is designated GS263-02+45. The region delimited in Fig. 2 by a dash-double dot boundary marks the region for which SGPS data were retrieved (see Sect. 4.2).

\section{Discussion}

\subsection{Supershell parameters}

Under the assumption of a spherically symmetric expansion, a HI supershell having a central velocity $V_{\mathrm{o}}$ and an expansion velocity $V_{\text {exp }}$ should depict, in a position-position diagram, a "diskring" pattern when observed at different radial velocities. At $V_{\mathrm{o}}$ the ring of $\mathrm{HI}$ emission attains its maximum diameter, while at extreme velocities (either approaching $\left(V_{\min }=V_{\mathrm{o}}-V_{\exp }\right)$ or receding $\left(V_{\max }=V_{\mathrm{o}}+V_{\exp }\right)$ the hydrogen emission should look like a "disk" (or "cap"). At intermediate velocities the radius of the HI ring shrinks as $V_{\max }$ (or $V_{\min }$ ) is approached. The expansion velocity is estimated as half of the total velocity range covered by the HI emission associated with the supershell $\left(V_{\exp }=0.5 \times\left(V_{\max }-V_{\min }\right)\right)$.

A visual inspection of Fig. 1 indicates that the central velocity of GS263-02+45 lies in the range from 44 to $46 \mathrm{~km} \mathrm{~s}^{-1}$. In order to derive a kinematic distance to GS263-02+45 the adopted central velocity was $V_{\mathrm{o}}=45 \pm 1 \mathrm{~km} \mathrm{~s}^{-1}$. Using both the linear fit and the power law fit of the galactic rotation model of Fich et al. (1989), a kinematic distance to GS263-02+45 of $5.3 \pm 1.0 \mathrm{kpc}$ is derived. The distance is a mean weighted value of the individual distances provided by both models. The weight of the individual distance determinations stems from the assumption of an uncertainty of $\pm 10 \mathrm{~km} \mathrm{~s}^{-1}$ due to non-circular motion (Burton 1988). The galactocentric distance of the supershell is $R_{\mathrm{g}}=10.6 \pm 0.6 \mathrm{kpc}$.

A least square fit of a circumference to the HI peaks defining GS263-02+45 is shown in Fig. 2 by a thin line. From this fit the supershell centre is $(l, b)=\left(262^{\circ} .6,-1^{\circ} .8\right)$ and its angular radius is about $3.6 \pm 0.4$. The coordinates of the supershell centre are accurate to \pm 0.3 . At a distance of $5.3 \pm 1 \mathrm{kpc}$, its angular dimensions translate to a linear diameter of $665 \pm 40 \mathrm{pc}$.

A closer look at Fig. 2 shows that the circumference is only a good first order approximation to the real shape of GS263$02+45$, as departures from a spherical symmetry are noticeable. To further illustrate this point, in Fig. 3 two cross-sections of the brightness temperature of GS263-02+45 as a function of the angular distance from its centre are shown. Each point in the crosssection represents a mean value of the HI brightness temperature emission along concentric rings spaced by $9^{\prime}$. In order to avoid most of the confusing effects arising from unrelated material, the numerical integration was performed between two ranges of position angles, namely from $58^{\circ}$ to $302^{\circ}$ (passing through $0^{\circ}$ ) (it will be referred to as the northern cross-section) and from $140^{\circ}$ to $221^{\circ}$ (it will be referred to as the southern cross-section). To obtain the cross-sections shown in Fig. 3 the corresponding minimum value of the HI brightness temperature was subtracted from the observed cross-cut value and latter normalised to the maximum remaining value. The maximum and minimum brightness temperature along the scans are $32.3 \mathrm{~K}$ and $5.3 \mathrm{~K}$ (northern cross-cut) and $22.8 \mathrm{~K}$ and $10.8 \mathrm{~K}$ (southern cross-cut), respectively. The mean weighted emission radius is $3.2 \pm 0.1$ 

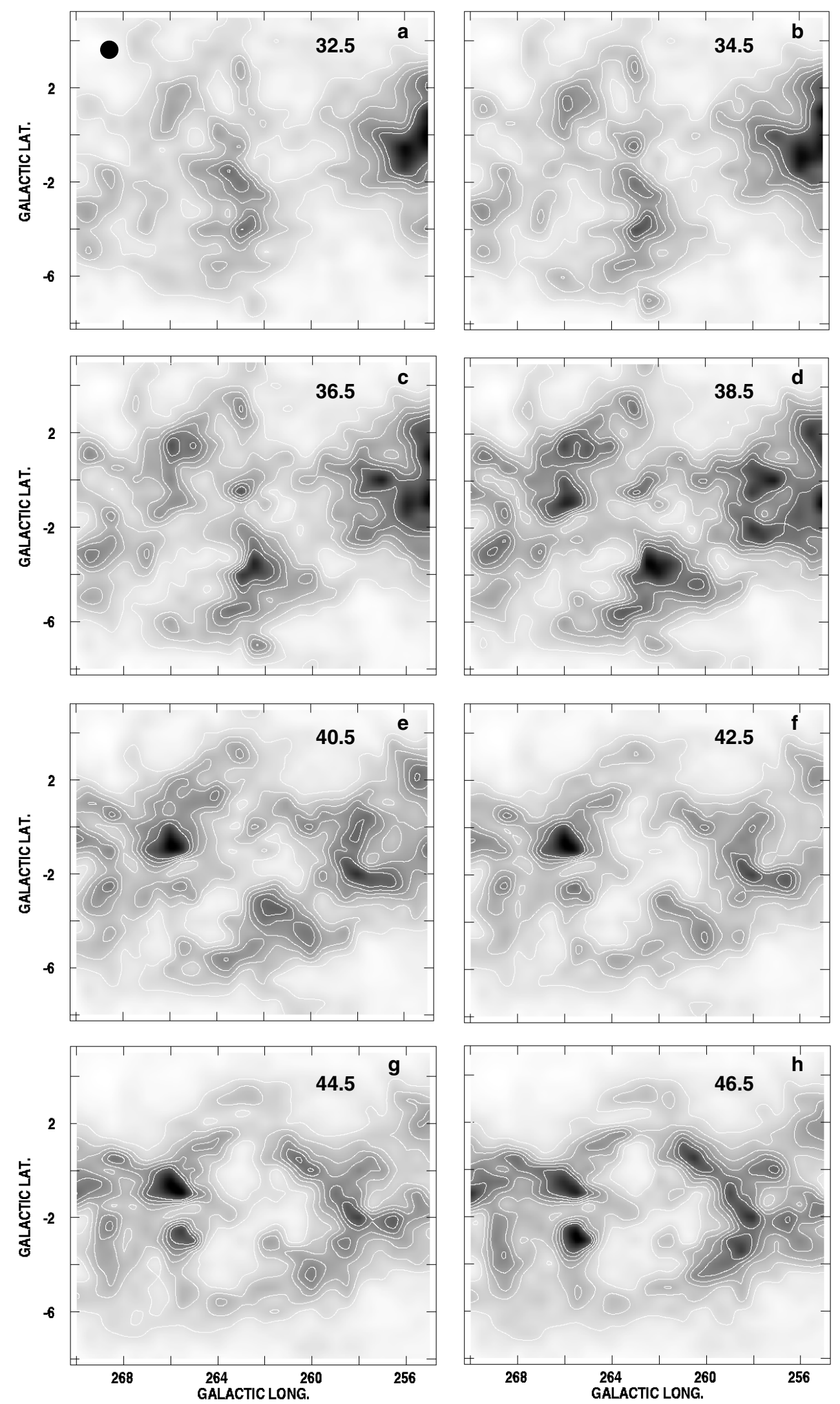

Fig. 1. Mosaic of HI mean brightness temperature in different velocity ranges. Each image is a mean of two individual images. The central velocity of each image is indicated at the inner top right corner. The filled circle drawn in the upper left corner of the HI image at 32.5 and $48.5 \mathrm{~km} \mathrm{~s}^{-1}$ indicates the angular resolution of the HI data. The lowest and highest contours are $15 \mathrm{~K}$ and $65 \mathrm{~K}$, respectively, while the contour spacing is $10 \mathrm{~K}$. The dark grey areas represent regions with high HI emissivity. 

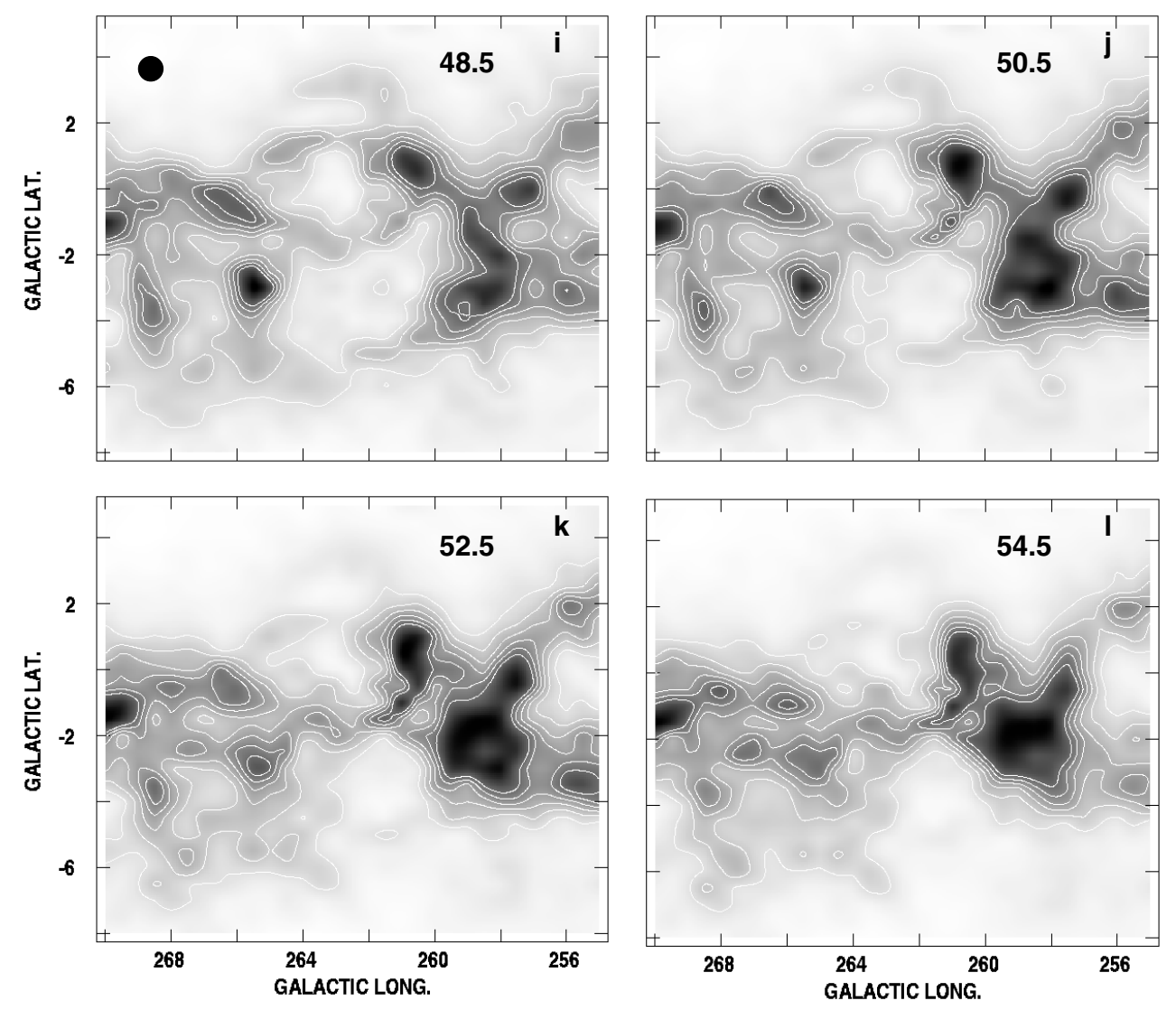

Fig. 1. continued.

(northern cross-cut) and $4.0 \pm 0.1$ (southern cross-cut), respectively. In both cases the angular HI shell thickness is about 1:3. At $5.3 \pm 1 \mathrm{kpc}$ the linear shell thickness is $120 \pm 25 \mathrm{pc}$.

The mass of the HI supershell is difficult to estimate in its entirety because of the uncertainty of whether some features are really part of the supershell, and because some parts of the shell may be confused with other HI galactic emission. Bearing in mind these caveats, the neutral hydrogen mass of GS263$02+45$ was derived from Fig. 2. In order to remove the unrelated galactic HI emission a warp plane was fitted through the hatched boxes shown in Fig. 2. The HI emission characterised by this plane is subtracted from the observed emission in order to produce a residual HI emission image mostly containing the $\mathrm{HI}$ emission related to GS263-02+45. In this residual image the lowest brightness temperature contour used to calculate the HI mass is $10 \mathrm{~K}$. Under the assumption of an optically thin $\mathrm{HI}$ emission and adopting $5.3 \pm 1 \mathrm{kpc}$ as the distance of GS263-02+45, the total HI mass is of the order of $M_{\mathrm{HI}}=1.2 \pm 0.6 \times 10^{6} M_{\odot}$. Assuming a helium abundance of $34 \%$ by mass, the total gaseous mass of GS263-02+45 is about $M_{\mathrm{t}}=1.7 \pm 0.8 \times 10^{6} M_{\odot}$.

The ambient density $\left(n_{\mathrm{o}}\right)$ of the medium into which GS263$02+45$ expanded is evaluated by uniformely distributing the supershell mass $\left(M_{\mathrm{t}}\right)$ over the volume of the structure. A value of $n_{\mathrm{o}} \sim 0.5 \mathrm{~cm}^{-3}$ is derived.

The expansion velocity plays a key role (along with the shell's total mass) at the time of evaluating the shell's total kinetic energy. At the beginning of this Section a procedure to determine $V_{\exp }$ was briefly outlined using position-position diagrams. In practice significant deviations from the idealized "disk-ring" pattern are to be expected because of inhomogeneities in the ISM. Moreover, due to confusion effects arising from the galactic background of neutral hydrogen emission, it is usually very dificult to identify the HI emission from gas moving at velocities close to either $V_{\max }$ or $V_{\min }$. Thus, from positionposition diagrams only a lower limit to $V_{\exp }$ can be found. Another procedure to determine $V_{\text {exp }}$ in expanding HI supershells was used by McClure-Griffiths et al. (2002). This method makes use of the Hi profile observed through the centre of the supershell. In our case, this profile is shown in Fig. 4a. There, the radial velocity of the $\mathrm{HI}$ emission related to the approaching and receding parts of the supershell can be determined. From Fig. $4 \mathrm{a}$ we derived $V_{\max } \sim 62 \mathrm{~km} \mathrm{~s}^{-1}$ and $V_{\min } \sim 33 \mathrm{~km} \mathrm{~s}^{-1}$. Thus, $V_{\text {exp }} \sim 14 \mathrm{~km} \mathrm{~s}^{-1}$. Due to the low latitude of the HI supershell $\left(b \sim-2^{\circ}\right)$ and the intricacies inherent to the galactic HI emission, one should verify the reliability of the expansion velocity derived following the method outlined above. To this end, an image showing the mean HI brightness temperature distribution in a velocity-galactic latitude diagram was constructed. This image is shown in Fig. $4 \mathrm{~b}$ for $262^{\circ} .5 \leq l \leq 263^{\circ} .5$. The strong HI emission observed at $V \sim+8 \mathrm{~km} \mathrm{~s}^{-1}$ arises from HI located in the solar neighbourhood, while the dash-dot ellipse approximately delineates the location of GS263-02+45 in this diagram. In Fig. $4 \mathrm{~b}$ at $b \sim-2^{\circ}$ the approaching gas is observed at $V_{\min } \sim 32-33 \mathrm{~km} \mathrm{~s}^{-1}$ and the receding gas at $V_{\max } \sim 61-62 \mathrm{~km} \mathrm{~s}^{-1}$. The velocity range of the observed HI emission and the behaviour of the HI related to GS263-02+45 is in agreement with an expansion velocity of $V_{\exp } \sim 14 \mathrm{~km} \mathrm{~s}^{-1}$. The total kinetic energy $\left(E_{\mathrm{k}}=0.5 \times M_{\mathrm{t}} \times V_{\exp }^{2}\right)$ of the expanding structure is $E_{\mathrm{k}} \sim(3.3 \pm 2.0) \times 10^{51} \mathrm{erg}$.

A reliable estimate of the age of GS263-02+45 is difficult to determine since there is no hint of the physical process that generated this supershell. To provide a rough order of magnitude, a dynamic age is derived by adopting the Weaver et al. (1977) 


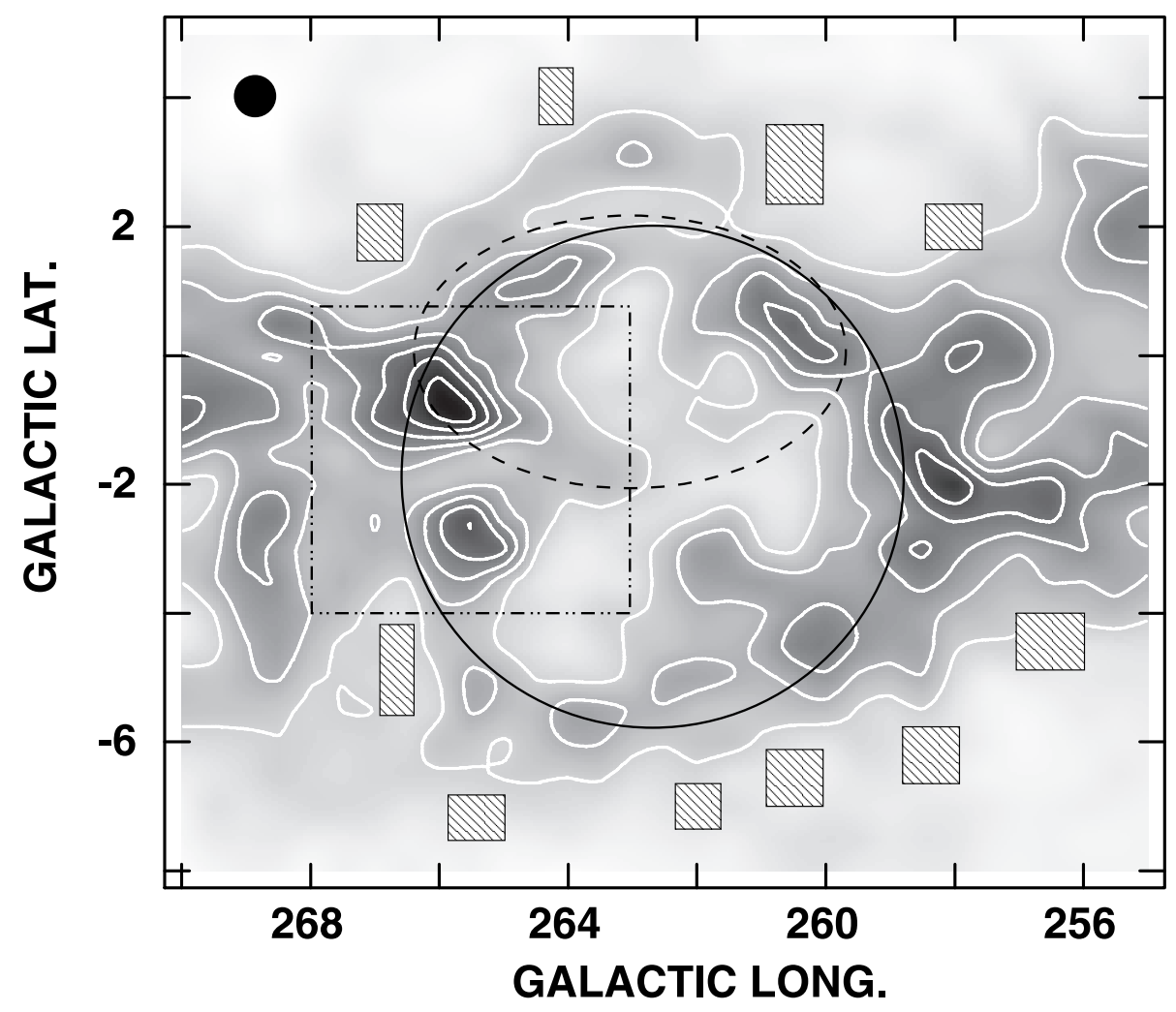

Fig. 2. Grey-scale representation of the mean brightness temperature in the velocity range 39 to $49 \mathrm{~km} \mathrm{~s}^{-1}$. The thin circumference represents the least square fit to the HI peaks defining GS263-02+45. The location of GSH263+00+47 is depicted by a dashed ellipse. The dash-double dot rectangle delimits the region shown in Fig. 5. The hatched rectangles mark the areas used to fit a warp plane (see text in Sect. 4.1).

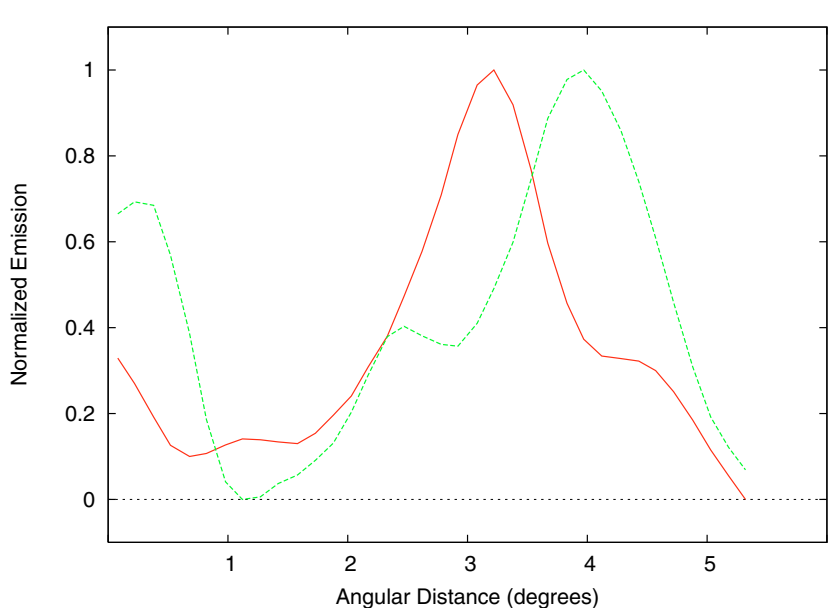

Fig. 3. Radial plots of the mean $\mathrm{HI}$ emission brightness temperature for the northern (solid line) and southern (dashed line) extremes of GS263-02+45 (see Sect. 4.1 for more details). Each data point represents the normalised mean brightness temperature value of a ring $9^{\prime}$ wide. The abscissae are mean angular distances, expressed in degrees, as measured from $(l, b)=\left(262^{\circ} .8,-1.8\right)$.

analytic solutions for a thin expanding shell created by a continuos injection of mechanical energy. Under these conditions a dynamic age of $\sim 1.3 \times 10^{7} \mathrm{yr}$ is derived.

The major source of error in the diameter, mass and kinetic energy of GS263-02+45 is the distance uncertainty. An error of $20 \%$ in the distance implies a $20 \%$ error in radius and $40 \%$ error in the derived mass. The uncertainty in the kinetic energy is at least $60 \%$ assuming a $10 \%$ error in the expansion velocity. The error in the derived ambient density $\left(n_{\mathrm{o}}\right)$ is about $100 \%$.

\subsection{Was the birth of Bochum 7 triggered by GS263-02+45?}

What evidence can be provided to support the hypothesis that the birth of Bochum 7 was triggered by the expansion of GS263-02+45? If this were the case, a few conditions should be met, namely: $a$ ) both objects should be located at comparable distances from the Sun; $b$ ) the HI supershell should be older than Bochum 7 ; c) since Bochum 7 is known to harbour O type stars, there should be hints of the interaction of both the stellar radiation field and the stellar winds of these massive stars with their surroundings; and $d$ ) the bulk mean proper motion of the stars of Bochum 7 should be directed away from the geometric centre of GS263-02+45. Based on the information provided in previous Sections, conditions $a$ ) and $b$ ) are, within the errors, fullfilled. For condition $c$ ), in Fig. 5 an enlargement of the region delimited by a dashed-double dot rectangle in Fig. 2 is shown. In Fig. 5 Bochum 7 appears projected onto a region of GS263$02+45$ depicting a HI emissivity lower than its surroundings. Within the latter, two HI minima also are seen. Bearing in mind that the HI emission defining the supershell has a high degree of small scale structure (see Fig. 1) it is very difficult to determine whether those HI minima are real or another manifestation of the small scale structure of GS263-02+45. For simplicity it will be assumed that Bochum 7 is projected onto a single large ovoid HI minimum. Adopting the $25 \mathrm{~K}$ contour level to define the outer boundary of this large minimum, it is found that the angular dimension of the major and minor semi-axis are $\sim 1^{\circ} .1$ and 0.45 , respectively. The equivalent angular radius $\left(R_{\mathrm{eq}}\right)$ of this ovoid structure is defined as the harmonic mean of the semi-axes. At the distance of GS263-02+45 it is found that $R_{\mathrm{eq}} \sim 65 \pm 15 \mathrm{pc}$.

Could the large HI minimum observed towards Bochum 7 have been created by the stellar winds of the most massive members of Bochum 7? Based on a stellar proper motion study carried out within a circle of $2^{\circ}$ in radius centred at the optical position of Bochum 7, Corti (2005) concluded that 

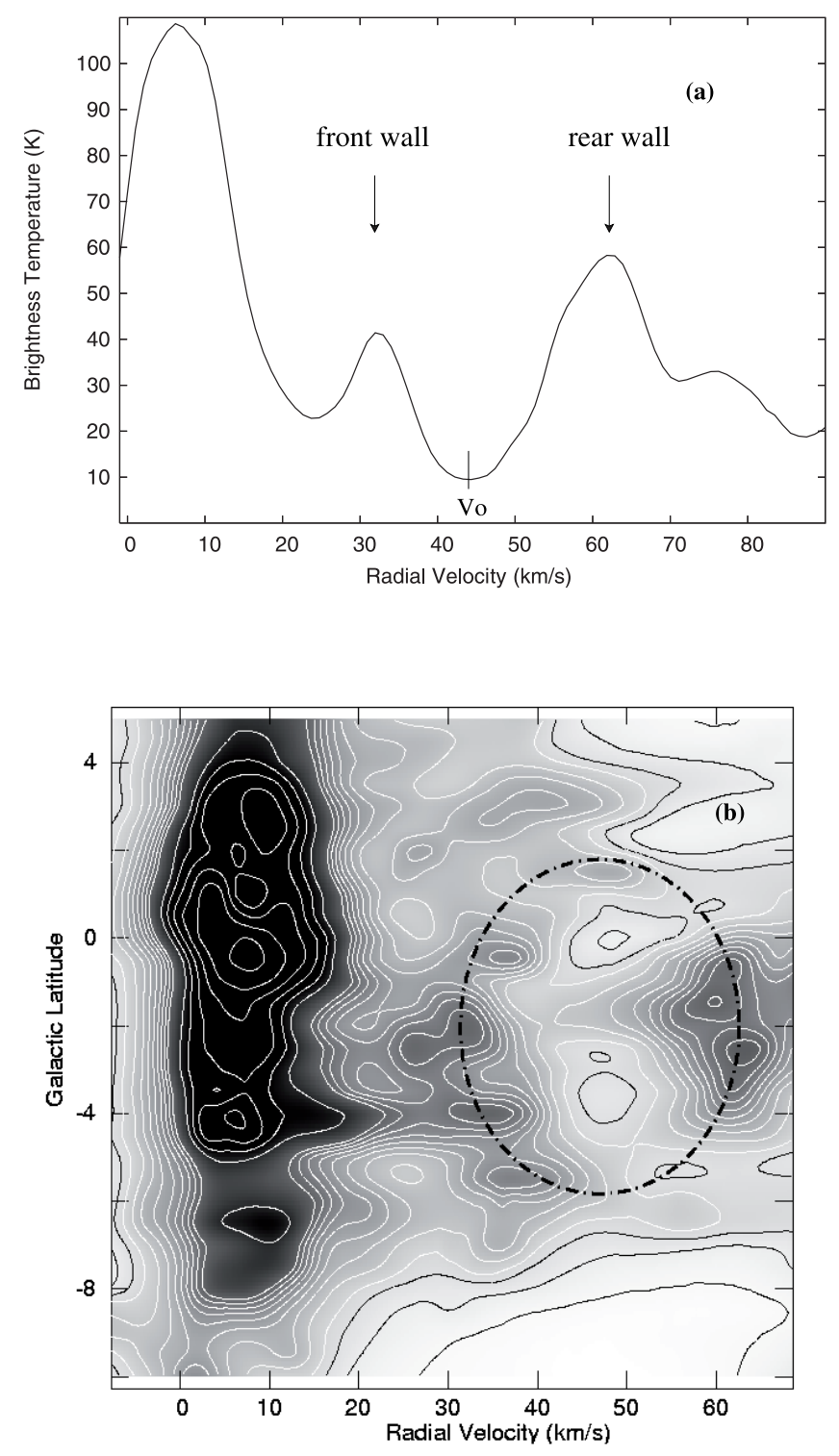

Fig. 4. a) HI spectrum obtained towards the centre of GS263-02+45. The approaching (front wall) and receding (rear wall) parts of the HI shell are identified. The expansion velocity is derived as half the velocity separation between the peak velocity of the front and rear shells. The central velocity is indicated by $V_{\mathrm{o}}$. b) Velocity-galactic latitude diagram of the mean brightness temperature distribution for $262^{\circ} .5 \leq l \leq 263^{\circ} .5$. The ridge of high HI emissivity depicted at $V \sim 8 \mathrm{~km} \mathrm{~s}^{-1}$ by dark grey tones arises from HI located within a few kiloparsecs from the Sun. The approximate location of GS263-02+45 in this diagram is shown by a dash-dot ellipse.

only 27 stars are candidates to be members of Bochum 7 . Seven of them (LS 1137 [O9-O9.5 V], LS 1140 [B0 III], LS 1146 [B1 III], CBN843648.6-460736 [B1-B1.5 V], CBN84505.0455648 [B2 V], CBN84505.0-460300 [B2.5-3 V], CBN84350.7455820 [B1.5 V] and CBN84518.8-454147 [B5 V]) have spectral types derived from spectroscopic observations (Corti 2005). In the above listing the prefix LS stands for stars listed in the LSS Catalogue (Stephenson \& Sanduleak 1971), while CBN indicates those stars identified by Corti et al. (2007). Following the guidelines of the IAU, CBN stars are identified using the capitalized first letters of the last names of the authors followed by

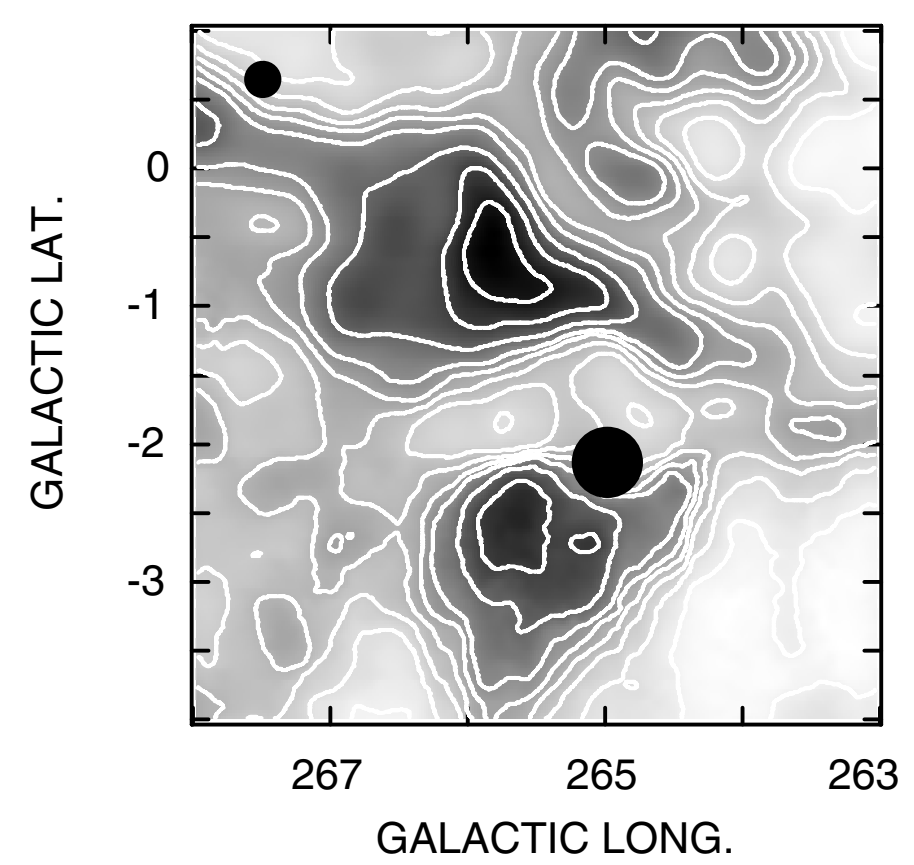

Fig. 5. Enlargement of the mean brightness temperature distribution around Bochum 7. This HI image was constructed using the SGPS data. The location of Bochum 7 is marked by a large filled black dot, while the angular resolution of the data is depicted by the filled dot seen on the upper left corner. Bochum 7 is projected onto a large region of low HI emissivity. The lowest and the highest brightness temperature contours are $5 \mathrm{~K}$ and $85 \mathrm{~K}$, respectively. The contour spacing is $5 \mathrm{~K}$ until $15 \mathrm{~K}$ and $10 \mathrm{~K}$ from there onwards. Regions of high HI emissivity are depicted by dark grey tonalities.

the object's celestial coordinates in the J2000.0 frame. For those candidate stars lacking spectroscopic observations (19 stars), spectral types ranging from $\mathrm{B} 1 \mathrm{~V}$ to $\mathrm{B} 8 \mathrm{~V}$ were derived by Corti et al. (2007). Additionally, relying on radial velocity measurements, three early type stars (LS 1135 [O6.5 V ((f)) + B0 V], LS 1131 [O7.5 V] and LS 1144 [O7.5 V]) may also belong to Bochum 7 (Corti 2005). In order to evaluate the possibility that the stellar winds of the members of Bochum 7 could have triggered the formation of the large HI minimum shown in Fig. 5, only those candidate stars of Bochum 7 having a spectral type on the main sequence earlier than $\mathrm{B} 0 \mathrm{~V}$ will be considered. This criterion is adopted based on the fact that the mechanical energy injected by an early B-type star is negligible compared to that of a star having a mid-O spectral type.

The mechanical energy $\left(L_{\mathrm{w}}\right)$ injected into the ISM by the stellar winds is given by

$L_{\mathrm{w}}=\frac{1}{2} \times \dot{M} \times v_{\infty}^{2}$

where $\dot{M}$ is the stellar mass-loss rate and $v_{\infty}$ is the wind velocity. Mass-loss rates were derived from

$\log \dot{M}=1.738 \times \log L-1.352 \times \log T_{\text {eff }}-9.547$

where $L$ is the stellar luminosity expressed in solar luminosity units and $T_{\text {eff }}$ is the effective stellar temperature expressed in $\mathrm{K}$. Equation (2) is the average relation for $\dot{M}$ in a sample of Galatic O stars studied by Lamers \& Leitherer (1993). According to these authors the standard deviation of individual mass-loss rates is $\sigma=0.23$. Wind velocities were derived from the theoretical relation of Leitherer et al. (1992)

$\log v_{\infty}=1.23-0.3 \times \log L+0.55 \times \log M+0.64 \times \log T_{\text {eff. }}$ (3) 
In Eq. (3), $M$ indicates the mass of the star (in solar mass units), while $L$ and $T_{\text {eff }}$ have the same meaning as before. The value of $v_{\infty}$ agree with observations to within $20 \%$. For a given spectral type, the basic stellar parameters $\left(L, M\right.$ and $\left.T_{\text {eff }}\right)$ were adopted from the values given by Vacca et al. (1996).

Based on the above, the current rate of mechanical energy input by the most massive members of Bochum 7 into the local ISM is $(3.1 \pm 3.0) \times 10^{36} \mathrm{erg} \mathrm{s}^{-1}$. Adopting the model for superbubble evolution that assumes a hot shocked-heated interior whose pressure drives the outer supershell growth (Dyson 1977) and a mean ISM density of $0.5 \mathrm{~cm}^{-3}$, the bubble produced by the most massive members of Bochum 7 should reach a radius $R_{\mathrm{b}} \sim 95 \pm 30 \mathrm{pc}$ after $4.5 \times 10^{6} \mathrm{yr}$ (the age of Bochum 7 ).

Bearing in mind the large uncertainties present in the determination of $R_{\mathrm{b}}$, it can be considered that within errors, the theoretical $\left(R_{\mathrm{b}}\right)$ and the observed $\left(R_{\mathrm{eq}}\right)$ radius of the bubble are in agreement.

In connection with condition $d$ ), after correcting the proper motions of the 27 star candidates for the motion of the LSR around the galactic centre and for the peculiar motion of the Sun with respect to the LSR, the mean peculiar proper motions of this set of stars along galactic longitude $\left(\mu_{l}\right)$ and galactic latitude $\left(\mu_{b}\right)$

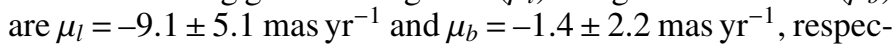
tively. Unfortunately, the large errors of these determinations, especially for $\mu_{l}$, cannot be used to place a reliable constraint on the direction of motion of Bochum 7 as a whole.

Summing up, it can be concluded that three of the four conditions specified at the beginning of Sect. 4.2 seem to support the hypothesis that the birth of Bochum 7 may be related to the evolution of GS263-02+45.

\section{Conclusions}

Making use of high sensitivity HI surveys carried out with single dish instruments, a new HI supershell has been found. This structure, designated as GS263-02+45, has its centre at $(l, b)=\left(262^{\circ} 8,-1.8\right)$. The neutral hydrogen associated with it is observable with the characteristic ring-like geometry of an expanding spherical shell along the velocity range from $\sim 38$ to $\sim 51 \mathrm{~km} \mathrm{~s}^{-1}$. The central velocity of GS263-02+45 is $45 \pm$ $1 \mathrm{~km} \mathrm{~s}^{-1}$ and its expansion velocity is about $\sim 14 \mathrm{~km} \mathrm{~s}^{-1}$. The kinematic distance of this supershell turns out to be $5.3 \pm 1 \mathrm{kpc}$. At this distance the total atomic gas content of GS263-02+45 is $\sim 2 \times 10^{6} M_{\odot}$, its mean radius is $\sim 330 \mathrm{pc}$ and the mean shell thickness is $\sim 120 \mathrm{pc}$.
Evidence has been found that the massive stars of the OBassociation Bochum 7 may be interacting (via their stellar winds) with the HI walls of GS263-02+45. This fact plus age and distance arguments may be interpreted as evidence in favour of a link between Bochum 7 and GS263-02+45.

Acknowledgements. This work was partially supported by the Agency ANPCyT through the grant PICT 03-14018-BID 1728/OC-AR. The authors would like to thank the anonymous referee for her/his comments and suggestions that helped to improve the paper. We also would like to thank Mr. M. Knight for a careful reading of the manuscript.

\section{References}

Arnal, E. M., Bajaja, E., Larrarte, J. J., Morras, R., \& Pöppel, W. G. L. 2000, A\&AS, 142, 35

Bajaja, E., Arnal, E. M., Larrarte, J. J., et al. 2005, A\&A, 440, 767

Burton, W. B. 1988, The structure of our Galaxy derived from observations of neutral hydrogen, Galactic and Extragalactic Radio Astronomy, 295

Corti, M. 2005, Ph.D. Thesis

Corti, M., Niemela, V., \& Morrell, N. 2003, A\&A, 405, 571

Corti, M., Bosch, G., \& Niemela, V. 2007, A\&A, 467, 137

de Blok, W. J. G., \& Walter, F. 2000, ApJ, 537, L95

Dyson, J. E. 1977, A\&A, 59, 161

Fich, M., Blitz, L., \& Stark, A. A. 1989, ApJ, 342, 272

Heiles, C. 1979, ApJ, 229, 533

Heiles, C. 1984, ApJS, 55, 585

Kalberla, P. M. W., Burton, W. B., Hartmann, D., et al. 2005, A\&A, 440, 775

Kim, S., Dopita, M. A., Staveley-Smith, L., \& Bessell, M. S. 1999, AJ, 118, 2797

Lamers, H. J. G. L. M., \& Leitherer, C. 1993, ApJ, 412, 771

Leitherer, C., Robert, C., \& Drissen, L. 1992, ApJ, 401, 596

McClure-Griffiths, N. M., Green, A. J., Dickey, J. M., et al. 2001, ApJ, 551, 394

McClure-Griffiths, N. M., Dickey, J. M., Gaensler, B. M., \& Green, A. J. 2002, ApJ, 578, 176

Oey, M. S., Watson, A. M., Kern, K., \& Walth, G. L. 2005, AJ, 129, 393

Perna, R., \& Raymond, J. 2000, ApJ, 539, 706

Price, S. D., Egan, M. P., Carey, S. J., Mizuno, D. R., \& Kuchar, T. A. 2001, AJ, 121,2819

Rand, R. J., \& Stone, J. M. 1996, AJ, 111, 190

Stanimirovic, S., Staveley-Smith, L., Dickey, J. M., Sault, R. J., \& Snowden, S. L. 1999, MNRAS, 302, 417

Stephenson, C., \& Sanduleak, N. 1971, Publ. Warner and Swasey Obs., 1, 1

Taylor, A. R. 1999, in New Perspectives on the Interstellar Medium, ed. A. R.

Taylor, T. L. Landecker, \& G. Joncas, ASP Conf. Ser., 168, 3

Tenorio-Tagle, G. 1981, A\&A, 94, 338

Vacca, W. D., Garmany, C. D., \& Shull, J. M. 1996, ApJ, 460, 914

Weaver, R., McCray, R., Castor, J., Shapiro, P., \& Moore, R. 1977, ApJ, 218, 377 